\title{
Smart Grid: An Overview
}

\section{Tamilmaran Vijayapriya $^{1 *}$, Dwarkadas Pralhadas Kothari ${ }^{2}$}

${ }^{1}$ Vellore Institute of Technology, Vellore, India; ${ }^{2}$ Vindhya Institute of Technology \& Science, Indore, India.

Email: "pvijayapriya@vit.ac.in,dpk0710@yahoo.com

Received December 30 ${ }^{\text {th }}, 2010$; revised June $7^{\text {th }}$, 2011; accepted June $13^{\text {th }}, 2011$.

\begin{abstract}
This paper briefly discusses evolution of Smart Grid development. Smart Grid is important as it will take us towards energy independence and environmentally sustainable economic growth. Growth of Smart Power Grid in India will slowly but surely take us towards fulfilling the dreams of former President Dr. A.P.J. Abdul Kalam, "Energy for all and Energy forever".
\end{abstract}

Keywords: Smart Grid, FACTS, ICT, Smart Homes

\section{Introduction}

In the present era, due to increased power demand to meet up the industrial requirements, the shortfalls in power generation have been attempted to mitigate between supply and demand through developments of National Grid connected systems where all the national power generation sources are connected to National grid and on the basis of the zonal requirement, the energy management is implemented. An "electricity grid" is not a single entity but an aggregate of multiple networks and multiple power generation companies with multiple operators employing varying levels of communication and coordination, most of which is manually controlled

With this concept, the earlier power shortage has been to some extent equated and is able to control the transmission losses and improve the transmission efficiency to some extent. This contrasts with 60 percent efficiency for grids based on the latest technology which may be the solution for the above problem: SMARD GRID TECHNOLOGIES.

To implement systematically the energy requirement for different zones, it necessarily requires a strategic program of distribution of energy. SCADA and other continuously monitoring systems though in vogue but for quick effective and efficient distribution of energy needs, a smart system which can take into account the requirements of the zones and the availability of energy from the different sources in the zones is required without human interference. Smart grids increase the connectivity, automation and coordination between these suppliers, consumers and networks that perform either long distance transmission or local distribution tasks
A smart grid is an umbrella term that covers modernization of both the transmission and distribution grids. The concept of a smart grid is that of a "digital upgrade" of distribution and long distance transmission grids to both optimize current operations by reducing the losses, as well as open up new markets for alternative energy production.

Some of the benefits of such a modernized electricity network include the ability to reduce power consumption at the consumer side during peak hours, called Demand side management; enabling grid connection of distributed generation power (with photovoltaic arrays, small wind turbines, micro hydro, or even combined heat power generators in buildings); incorporating grid energy storage for distributed generation load balancing; and eliminating failures such as widespread power grid cascading failures. The increased efficiency and reliability of the smart grid is expected to save consumers money and help reduce $\mathrm{CO}_{2}$ emissions. Governments increasingly focus on energy security, investing in the Smart Grid could be used to reduce dependence on non-domestic energy sources. It could also make the grid more resistant to military or terrorist attacks, by physical or digital means

Smart grid is referred to by other names including "Smart Electric Grid”, “Smart Power Grid”, "Intelligrid”, and "Future Grid" [1].

\section{Smart Grid}

A Smart Grid is an electricity network that can intelligently integrate the actions of all users connected to it generators, consumers and those that do both-in order to efficiently deliver sustainable, economic and secure 
electricity supplies [2]. A Smart Grid employs innovative products and services together with intelligent monitoring, control, communication, and self-healing technologies to:

- better facilitate the connection and operation of generators of all sizes and technologies;

- allow consumers to play a part in optimizing the operation of the system;

- provide consumers with greater information and choice of supply;

- significantly reduce the environmental impact of the whole electricity supply system;

- deliver enhanced levels of reliability and security of supply.

\subsection{Aims of the Smart Grids-The Vision}

- Provide a user-centric approach and allow new services to enter into the market;

- Establish innovation as an economical driver for the electricity networks renewal;

- Maintain security of supply, ensure integration and interoperability;

- Provide accessibility to a liberalized market and foster competition;

- Enable distributed generation and utilization of renewable energy sources;

- Ensure best use of central generation;

- Consider appropriately the impact of environmental limitations;

- Enable demand side participation (DSR, DSM);

- Inform the political and regulatory aspects;

- Consider the societal aspects [2].

\subsection{Pathways to a Smart Grid}

Movement from static infrastructure to a flexible power grid with enhanced observability, controllability and pro- cess efficiency [3], From T.

\subsection{Components of Smart Grid [4]}

The basic components of SmartGrid is as shown in Figure 1.

\subsection{The Key Challenges for Smart Grids}

- Strengthening the grid-ensuring that there is sufficient transmission capacity to interconnect energy resources, especially renewable resources;

- Moving offshore-developing the most efficient connections for offshore wind farms and for other marine technologies;

- Developing decentralized architectures_-enabling smaIller scale electricity supply systems to operate harmoniously with the total system;

- Communications-delivering the communications infrastructure to allow potentially millions of parties to operate and trade in the single market;

- Active demand side - enabling all consumers, with or without their own generation, to play an active role in the operation of the system;

- Integrating intermittent generation-finding the best ways of integrating intermittent generation including residential microgeneration;

- Enhanced intelligence of generation, demand and most notably in the grid;

- Preparing for electric vehicles-whereas Smart Grids must accommodate the needs of all consumers, electric vehicles are particularly emphasized due to their mobile and highly dispersed character and possible massive deployment in the next years, what would yield a major challenge for the future electricity networks [2].

The earliest, and still largest, example of a smart grid is the Italian system installed by Enel S. p. A. of Italy.

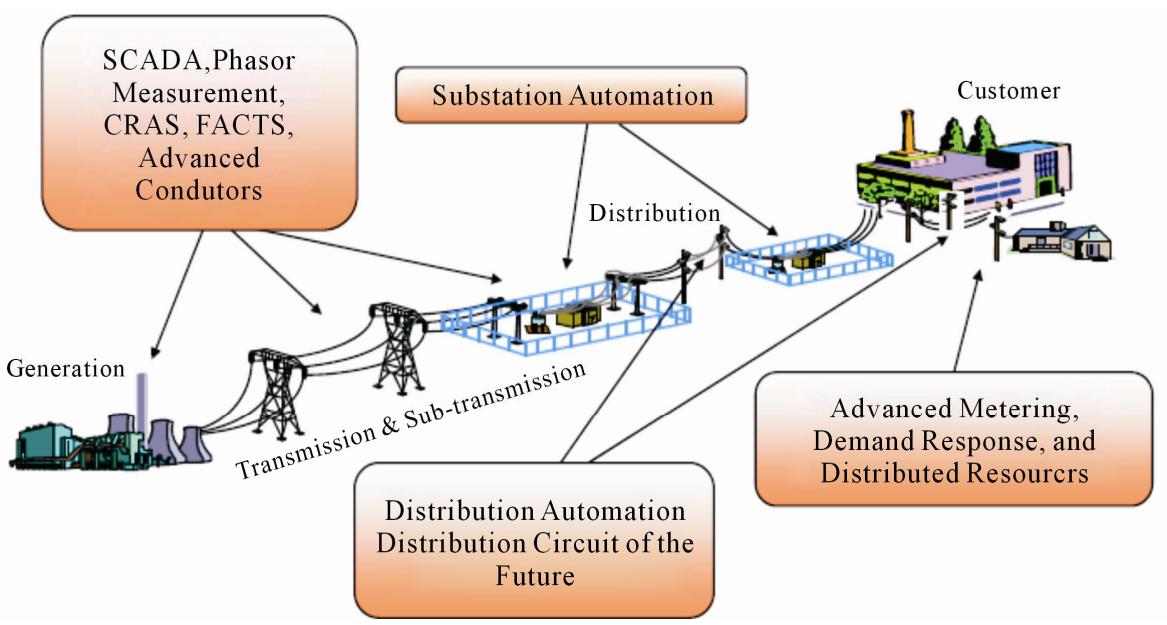

Figure 1. Components of smart grid. 
Completed in 2005, the Telegestore project was highly unusual in the utility world because the company designed and manufactured their own meters, acted as their own system integrator, and developed their own system software. The Telegestore project is widely regarded as the first commercial scale use of smart grid technology to the home.

\subsection{A Model Set Up [4]}

A model set up of Smart Grid including smart generation, smart transmission, smart storage, smart sensors to isolate the fault is given in Figure 2.

\section{Action Plans}

In order for the Smart Grids Vision to become a reality, a plan of actions is needed to allow the many facets of technical, regulatory, environmental and cultural issues to be addressed in an optimized manner. This will provide a coherent deployment of research and development results, integrated with existing infrastructure and technology, delivering early benefits, while maintaining the steady progress and evolution towards the main goals [2].

\subsection{Optimizing Grid Operation and Use}

In order to manage the ever increasing demands for energy trading and security of supply, the existing transmission and distribution networks require improved integration and coordination. To control electric power flows across state borders, advanced applications and tools, that are already available today, should be deployed to manage the complex interaction of operational security and trading and to provide active prevention and remedy of disturbances.

Key elements and priority components

- Wide Area Monitoring (WAM) and Wide Area Control (WAC) systems with regulation of static VAR compensators, optionally in a closed loop, to maximize the use of available transmission capacity while reducing the likelihood of disturbances;

- Distributed state estimators for large synchronous areas with real-time power system security assessment and optimized dispatching with dynamic constraints;

- System operators' staff training covering traditional issues (e.g. power system control) and emerging issues (e.g. electricity market and regulation);

- Coordinated ancillary services, including integration of balancing markets and coordination of reserves throughout the grids/control areas - the integration of balancing markets is of particular importance both, for enhanced power system security and for improved market liquidity;

- Steady state and dynamic (transient) simulators with modeling of Renewable Energy Sources and non-linear devices;

- Coordinated operation of power flow control systems (FACTS, phase shifters, etc.) with devices for automatic counter measures/system defense. These appli-

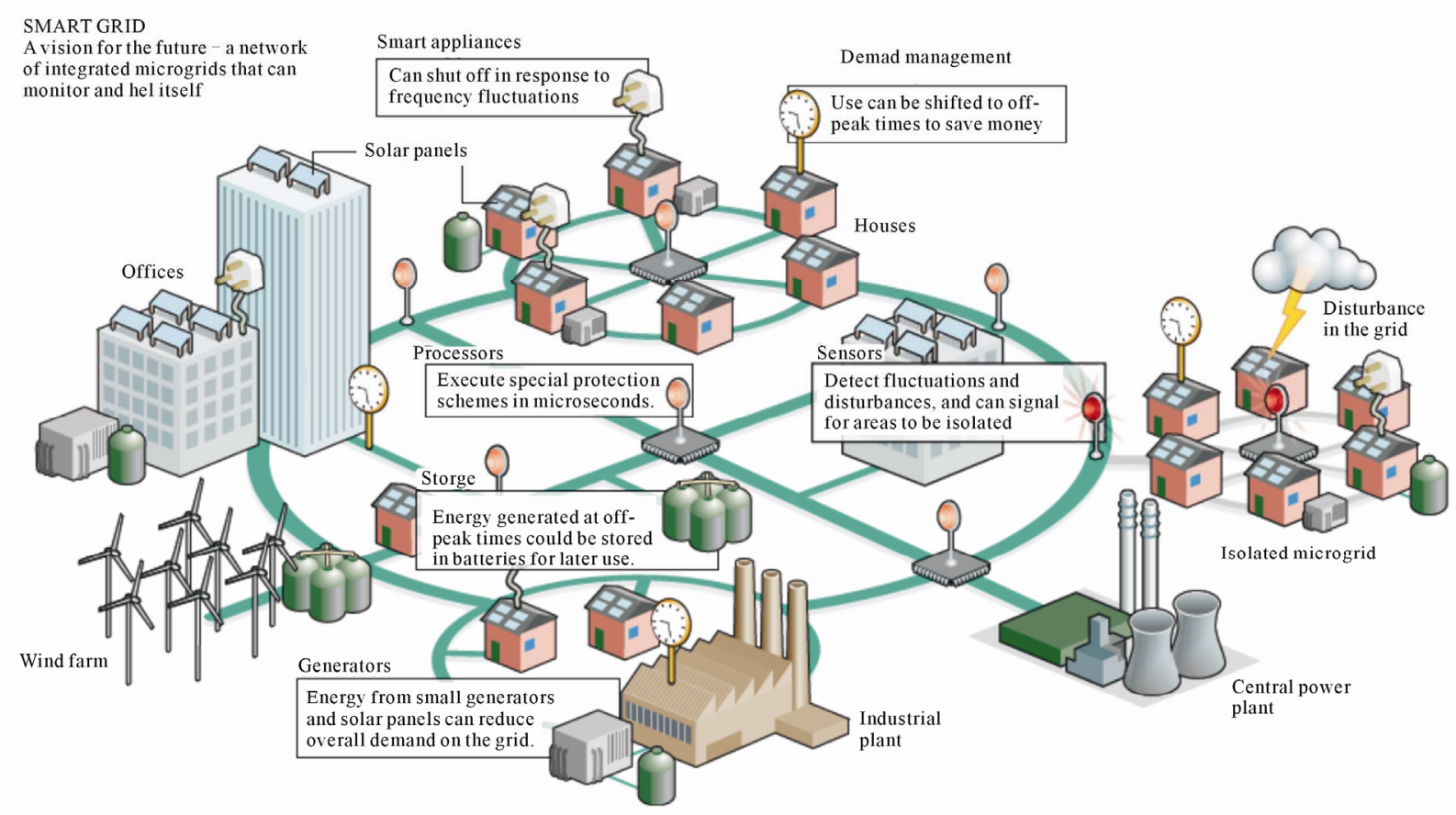

Figure 2. A model set up of smart grid network. 
cations exist in component form today but, unless encouraged, not many more will be deployed in the short time. Further work is urgently required to understand how to deploy and validate these solutions in "closed-loop" operation;

- Regulatory issues of relevance for the defined targets should be re-considered to ensure innovative technological solutions are adequately promoted and deployed.

\subsection{Optimizing Grid Infrastructure}

New and efficient asset management solutions for the transmission and distribution grids are required, as well as coordinated and coherent grid infrastructure planning should be done. Rather than being only deterministic, coordinated planning should be based on scenarios and include the necessary elements of risk management in order to cope with the increased volatility and uncertainty in location and size of generation and growing intermittent generation.

\section{Key elements and priority components}

- Expanding the grids (notably transmission) with new infrastructure (e.g. HVDC) will depend on accelerating permitting procedures and making them much more efficient than today;

- New overhead line configurations to increase capacity and reduce electromagnetic fields are required;

- Refurbishment/enforcement of the existing high voltage lines by innovative network assets including superconductivity technology;

- New asset management and grid planning methods for transmission and distribution;

- Development of systems and components to maintain power quality at acceptable levels while encouraging the integration of new types of generators.

\subsection{Integrating Large Scale Intermittent Generation}

Large-scale forms of generation, e.g. wind farms and in the future (concentrated) solar thermal generation, require networks to enable efficient collection of the power generated and enable system balancing, either by energy storage, conventional generation or by demand side participation [5]. Off-shore wind energy needs marine power collection networks and reinforcement of the terrestrial networks. This deployment priority is hence also about promoting and fostering the large-scale integration of renewable energy resources in a manner that meets the requirements of grid security while considering economic efficiency.

Key elements and priority components

- Technically viable and commercially affordable solutions for offshore networks for collection of wind power;

- Grid connection from offshore networks should consider security and quality of supply, economy and environmental sustainability;

- Transnational and cross-border grid re-enforcements should be considered. The present long licensing procedures should be shortened;

- Solutions should be developed to allow for efficient and secure system operation of future grids with significant intermittent generation, heavy bulk power and/or not easily dispatchable.

\subsection{Information and Communication Technology}

This is about defining the tasks and implementing the necessary standards for Information and Communication Technology (ICT) solutions in future Smart Grids. The application of ICT is a pre-requisite for data exchange between the different market players in the electricity supply chain and for the secure, economic and environmentally benign operation of Smart Grids [6].

Today ICT is applied at the transmission and subtransmission level and ends at the bus-bars of the subtransmission $(110 \mathrm{kV}) /$ medium voltage substations. Different standard protocols at various voltage levels and for different kinds of equipment are used. By large, the medium and low voltage levels are characterized by limited ICT, for economic reasons. Standardized, open information models and communication services for all data exchange within the whole electricity supply chain and electric power supply system are needed.

Different ICT technologies should be investigated and tested on site with the goal towards the introduction of ICT into the distribution level relying on the existing communication infrastructure (radio, power line, copper or fibre optics), applied in a cost effective way. The challenge will be to coordinate all these data bases through one overlaying data warehouse based on common information models (CIM). The data warehouse concept interconnects to all other data bases ensuring this way the necessary data consistency.

Key elements and priority components

- Simple, robust, secure and flexible communication infrastructure to allow monitoring, management, control and dispatching operations at all levels down to the distribution and customers;

- Common information and data models for all information building blocks, in order to ensure consistent database management, need to be defined at all levels of the power system and electricity supply chain;

- Well functioning ICT solutions are essential for maintaining the security of supply and for the efficient interaction of the market players; 
- A truly competitive situation for all kinds of products relying on multi-vendor strategies can only be achieved with well defined and standardized ICT solutions.

\subsection{Active Distribution Networks}

Transmission networks have always provided a balancing and management role in the electric power supply chain, whereas distribution networks have been designed to be passive ("fit-and forget") in operation. The challenge is now to provide many of the services found in transmission grids, such as power flow and constraint management, contingency analysis and balancing in distribution networks [7]. This is required not just because of the increaseing deployment of distributed generation, but also because of emerging intelligent building services in both residential and commercial premises, the need for utilizing local generation to support the local network at times of stress on the main grid and because of the anticipated future wide usage of electrical transportation vehicles.

Distribution networks will need to be able to respond or adapt in real-time to the complex interactions of all of these challenges and provide enhanced information to various actors to enable the real-time trading of the various services being provided.

Key elements and priority components

- An active network requires effective and coherent visibility of the various devices connected to it in order to allow timely decision making and information flow;

- Centralized manual control needs to be replaced by a distributed control architecture which will be coordinated and integrated into existing control methodologies in order to take advantage of the intelligence that will enhance the networks of the future;

- It is necessary to ensure compatibility of all functions and devices also during the transition from the present to the future active distribution grids;

- Besides on-line control and management, the active distribution network will introduce new functionalities, enabled by new tools and solutions relying on dynamic and multifaceted optimization [8]. Modeling of uncertainties in planning and operation required to achieve that, will build upon:

- Standardization of the data models and communication protocols to ensure minimum overhead and capacity to expand and encompass future requireements;

- Communication systems capable of coping with the needs in terms of capacity, reliability and costs induced by the new functions.

\subsection{New Market Places, Users and Energy Efficiency}

Diminishing of the differences between transmission and distribution in areas such as ancillary services, grid connection and access, but also quality and security of supply is one of the important characteristics of the whole Smart Grids concept. At the same time, such "democratization" and "decentralization" requires enhanced and strengthened control and management. This is not only necessary to operate the grid securely-adequate control and management solutions are also required to deploy a number of new and emerging concepts successfully and effectively such as the Virtual Power Plants and end-user energy management concepts.

In order to meet future customer needs, a range of new market participants will evolve. Besides transparent and nondiscriminatory grid access and connection for all grid users (generation and demand) this deployment priority is also relying on the necessary technologies in the so called "last mile" of the Smart Grids

The customers' needs, interests and benefits are clearly the focus of this deployment priority. Moreover, white goods in houses will contribute to the efficiency of the electricity networks in the future but only if there is a coordinated activity between the network, the smart meter, the user and the manufacturer of the goods. Revenue streams will need to be in place for such developments to take place.

Key elements and priority components

- Innovative Customer Interface Devices as bidirectional smart communicators between the customers and the market.

- To give the customer choice in energy supply, it is necessary to develop solutions to increase and optimize information related to energy consumption, improving the interaction between customers and market players;

- Such devices shall be able to provide the relevant energy information stored in digital or electronic meters to stimulate the consciousness and generate a virtuous new behavior toward energy savings, increasing end-users energy efficiency;

- Such devices can also work as "energy data providers" for all the smart appliances installed in house, in order to enable load management services.

- Intelligent Smart Home Controller, providing information on patterns of behavior, useful for raising awareness of energy consumption and to foster efforts towards real energy conservation/savings.

- Encouraging the customers' active role requires advanced ICT tools able to manage the complexity of multiple inputs, take consequent intelligent actions and provide easy and flexible interaction between the customers and the system;

- The Smart Home Controller should represent the 


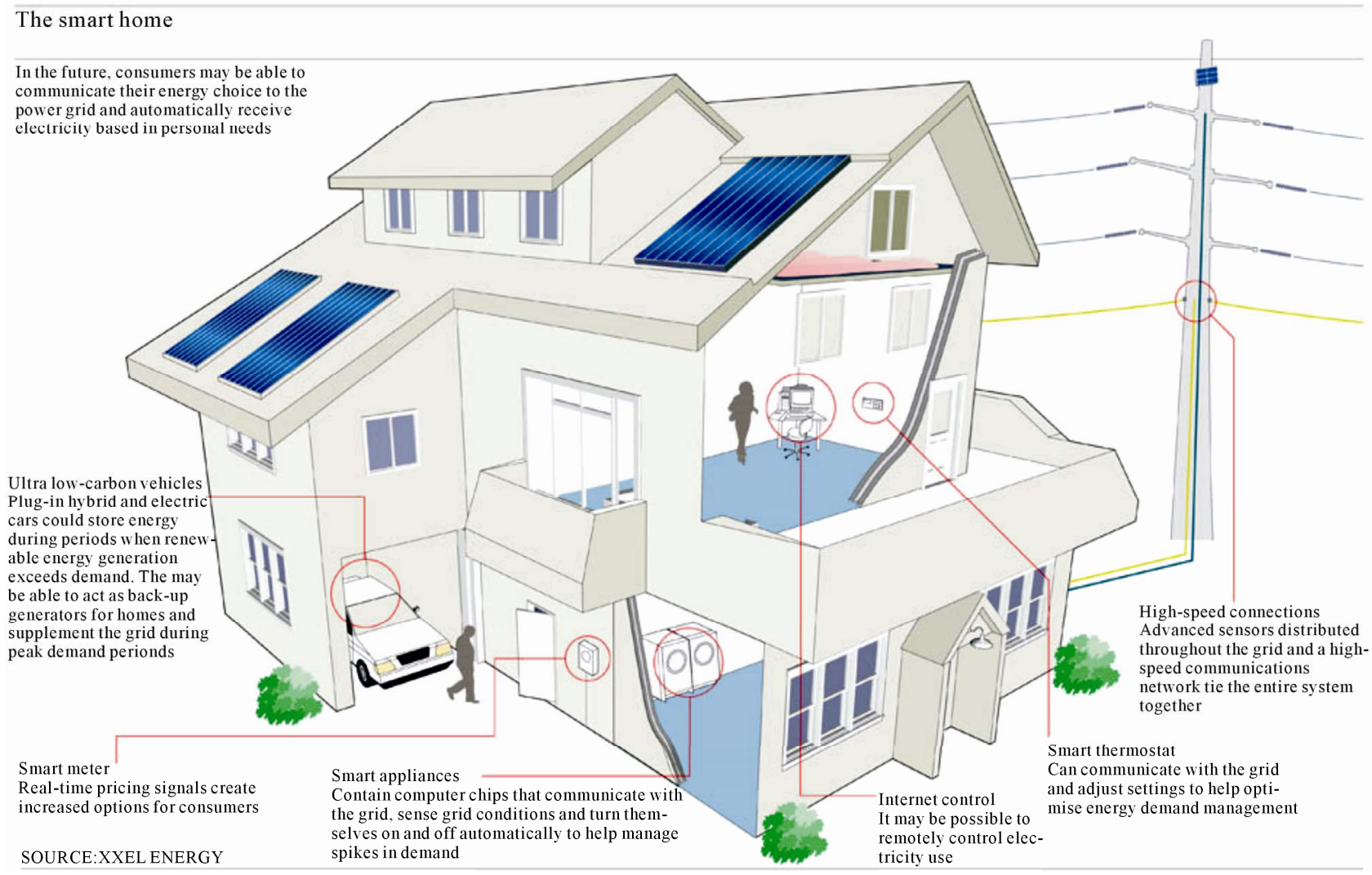

Figure 3. A smart home model.

control point and counterpart to smart meters. They will interact closely in order to exchange data;

- The customers' active role will be focused on setting the rules and priorities of energy use in respect of availability and cost while the daily operations, information and communication.

\section{A Conceptual Smart House}

Smart home is becoming a reality in the developed world with Energy efficiency and reduction in Carbon footprints riding high on the agenda of most the Governments and States. A model set of it is as shown in Figure 3. The technological advances in Smart meters, internet communication and smart appliances has made it achievable and sustainable.

The list of Smart devices include:

- Electricity Smart meter;

- Gas Smart meter (more likely to communicate via Electricity Smart Meter);

- Water Smart meter (more likely to communicate via Electricity Smart Meter);

- Home Automation Gateway (more likely to communicate over internet and radio);

- Home Smart Appliances (more likely to communicate over radio);
- Heating, Ventilation and Air-conditioning;

- Fridge;

- Washing Machine;

- Audio and Video;

- Lightning;

- Robotics.

\section{Conclusions}

This paper has dealt with the evolution of Smart Power Grid System. It is still in it nascent stage. The whole power community is busy now in understanding and developing smart power grid system which is no longer a theme of future. This introductory paper is a small but a very vital step towards achieving the ultimate goal of making a "National Grid" a reality.

\section{REFERENCES}

[1] M. Weedall, “BPA Smart Grid Overview,” Energy and Communications, Washington House Technology, January 22, 2000.

http://www.pnwer.org/portals/9/BPA smart grid.pdf

[2] European Technology Platform, "SMART GRIDS"Strategic Deployment Document for Europe's Electricity Networks of the Future, September 2008. http://www.smartgrid.eu/documents/smart 
grids_SDD_Final_April2010.pdf

[3] U. Niehage, "Pathways to a Smart Grid," Power Transmission and Distribution, Siemens, 8 November 2007. http://www.smartgrid.eu/documents/2ndGA/Niehage.pdf

[4] S. Rahman, "The Smart Grid and Its Impact on the Integration of Distributed Energy Resources," Southeast University, Nanjing, 2 April 2009.

[5] P. F. Ruiz, “Towards Smart Power Networks,” Lessons Learned from European Research FP5 Projects-European Commission in 2005, 2005. http://ec.europa.eu/research/energy.pdf/towards

[6] IBM, "Smart Grid Overview-IBM,” International Exhibition and Conference-Gridtech 2009, New Delhi, January 29-30, 2009.

[7] D. P. Kothari and I. J. Nagrath, "Modern Power System Analysis,” 3rd Edition, McGraw Hill, New York, 2006.

[8] D. P. Kothari and I. J. Nagrath, "Power System Engineering," 2nd Edition, Tata McGraw Hill, New Delhi, 2007. 